Research Article

\title{
The Oncogenic Role of CENPA in Hepatocellular Carcinoma Development: Evidence from Bioinformatic Analysis
}

\author{
Yuan Zhang $\mathbb{D}^{1}$, Lei Yang $\mathbb{D},{ }^{2}$ Jia Shi $\mathbb{D},{ }^{1}$ Yunfei Lu $\mathbb{D}^{1},{ }^{1}$ Xiaorong Chen $\mathbb{D}{ }^{1}$ \\ and Zongguo Yang ${ }^{1}{ }^{1}$ \\ ${ }^{1}$ Department of Integrative Medicine, Shanghai Public Health Clinical Center, Fudan University, Shanghai 201508, China \\ ${ }^{2}$ Department of Acupuncture and Moxibustion, Dongzhimen Hospital, Beijing University of Chinese Medicine, Beijing 100007, China \\ Correspondence should be addressed to Xiaorong Chen; chenxiaorong@shphc.org.cn \\ and Zongguo Yang; yangzongguo@shphc.org.cn
}

Received 27 September 2019; Revised 14 March 2020; Accepted 20 March 2020; Published 8 April 2020

Academic Editor: Ali A. Khraibi

Copyright ( 2020 Yuan Zhang et al. This is an open access article distributed under the Creative Commons Attribution License, which permits unrestricted use, distribution, and reproduction in any medium, provided the original work is properly cited.

Objective. This study is aimed at investigating the predictive value of CENPA in hepatocellular carcinoma (HCC) development. Methods. Using integrated bioinformatic analysis, we evaluated the CENPA mRNA expression in tumor and adjacent tissues and correlated it with HCC survival and clinicopathological features. A Cox regression hazard model was also performed. Results. CENPA mRNA was significantly upregulated in tumor tissues compared with that in adjacent tissues, which were validated in The Cancer Genome Atlas (TCGA) and Gene Expression Omnibus (GEO) series (all $P<0.01$ ). In the Kaplan-Meier plotter platform, the high level of CENPA mRNA was significantly correlated with overall survival (OS), disease-free survival (DFS), recurrence-free survival (RFS), and progression-free survival (PFS) in HCC patients (all $\log \operatorname{rank} P<0.01$ ). For validation in GSE14520 and pan-TCGA dataset, HCC patients with CNEPA mRNA overexpression had poor OS compared with those with low CENPA mRNA (log rank $P=0.025$ and $P<0.0001$, respectively), and those with high CENPA had poor DFS in TCGA ( $\log$ rank $P=0.0001$ ). Additionally, CENPA mRNA were upregulated in HCC patients with alpha-fetoprotein (AFP) elevation, advanced TNM stage, larger tumor size, advanced AJCC stage, advanced pathology grade, and vascular invasion (all $P<0.05$ ). A Cox regression model including CENPA, OIP5, and AURKB could predict OS in HCC patients effectively $(\mathrm{AUC}=0.683)$. Conclusion. Overexpressed in tumors, CENPA might be an oncogenic factor in the development of HCC patients.

\section{Introduction}

As a histone $\mathrm{H} 3$ variant of centromeric nucleosomes, centromere protein A (CENPA) is required to guarantee kinetochore for proper chromosome segregation assembly [1, 2]. Resulting from artificial overexpression, CENPA mislocalization could potentially generate ectopic kinetochores or weaken native kinetochores, leading to aberrant chromosomal segregation and instable genome [3-5]. An aberrant expression of CENPA and defects in CENPA function result in disrupted genome integrity, abnormal cell division, and thereby cancer [6-8]. Previous reports indicated that nonphysiological incorporation of CENPA may promote human tumorigenesis [9-11].
Previous publications revealed that CENPA was aberrantly overexpressed in hepatocellular carcinoma (HCC) tumor tissues. CENPA promoted HCC cell proliferation both in vitro and in vivo. siRNA-induced CENPA depletion blocked cell cycle progression and assisted apoptosis in HCC cells via numerous genes implicated in cell cycle control and apoptosis. CENPA overexpression was correlated with HBsAg positive status, advanced histological grade, high Ki-67 index, and P53 immunopositivity [12, 13]. However, the clinical predictive value of CENPA in HCC prognosis has not been well illustrated.

This study aimed at investigating CENPA expression in HCC tissues and correlating it with HCC survival and clinicopathological characteristics, in the attempt to validate the 
TABLE 1: Details of GEO series included in this analysis.

\begin{tabular}{lcccc}
\hline GEO series & Contributor(s) & Tumor & Nontumor & Platform \\
\hline GSE14520 & Roessler S et al., 2009 & 222 & 212 & Affymetrix Human Genome U133A 2.0 Array/Affymetrix \\
GSE36376 & Park CK, 2012 & 240 & 193 & HT Human Genome U133A Array \\
GSE60502 & Kao KJ, 2014 & 18 & 18 & Illumina HumanHT-12 V4.0 expression beadchip \\
GSE74656 & Yin H, 2015 & 5 & 5 & $\begin{array}{c}\text { Affymetrix Human Genome U133A Array } \\
\text { GSE77314 }\end{array}$ \\
\hline
\end{tabular}

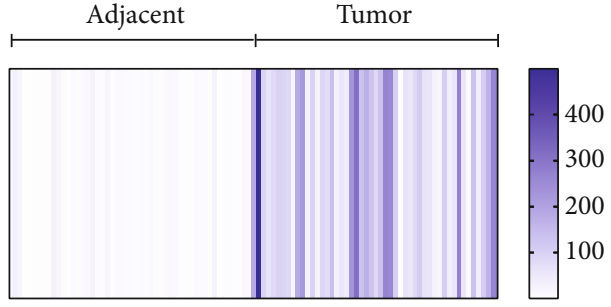

(a)

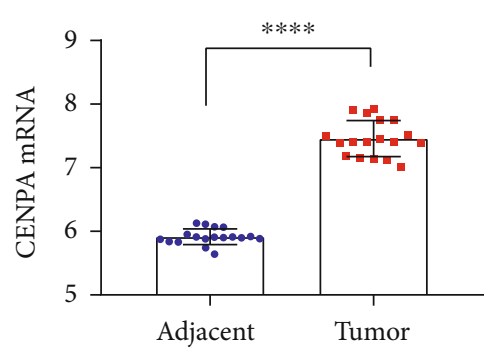

(d)

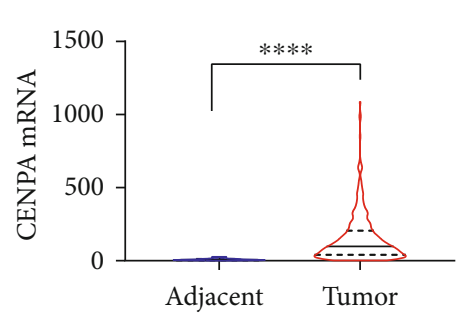

(b)

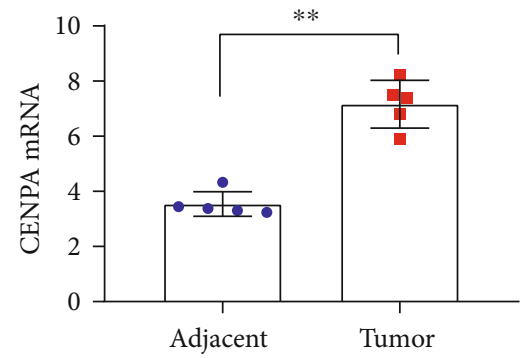

(e)

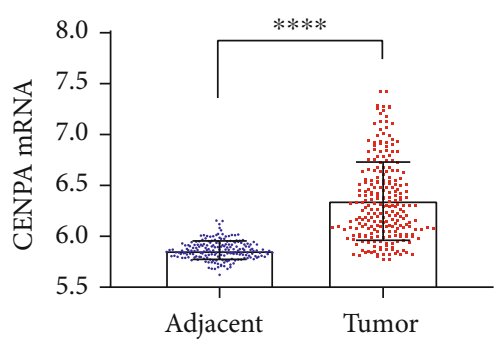

(c)

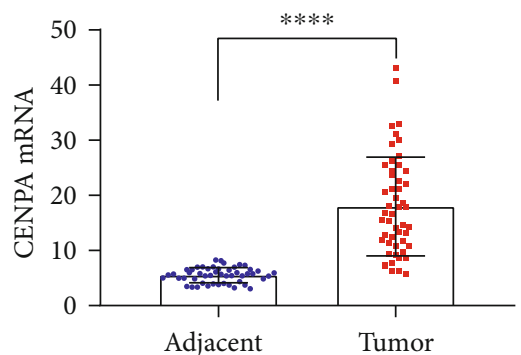

(f)

FIGURE 1: CENPA mRNA expression between tumor and adjacent tissues in HCC patients. CENPA mRNA was significantly upregulated in tumor tissues in 50 paired tumor and adjacent tissues from (a) TCGA profile, (b) entire TCGA profile $(P<0.0001)$, (c) GSE36376 series $(P<0.0001)$, (d) GSE60502 series $(P<0.0001)$, (e) GSE74656 series $(P<0.01)$, and (f) GSE77314 series $(P<0.0001)$.

oncogenic roles of CENPA and evaluate its predictive value in HCC aggressiveness.

\section{Materials and Methods}

2.1. Microarray Data. The microarray profiles including GSE36376, GSE60502, GSE74656, and GSE77314 were obtained from Gene Expression Omnibus (GEO, https:// www.ncbi.nlm.nih.gov/geo/). GSE14520 [14, 15] was used to validate the CENPA mRNA expression and its relationship with HCC outcomes. The details of these GEO series were summarized in Table 1. CENPA protein expression detected by an immunohistochemical assay was investigated in the Human Protein Atlas (HPA, https://www.proteinatlas .org/) database [16-18].

Expression and clinical data of liver hepatocellular carcinoma in The Cancer Genome Atlas (TCGA) PanCancer Altas was downloaded from cBioPortal for cancer genomics (http://www.cbioportal.org/) $[19,20]$.
2.2. Survival Analysis. Survival analysis of CENPA in HCC patients was conducted in a Kaplan-Meier plotter [21, 22]. The database is processed by a PostgreSQL server, which integrates both gene expression and clinical data. Patient samples were divided into two groups by a median cutoff of CENPA (RNAseq ID: 1058) to analyze the prognostic value. Two patient groups were compared by the Kaplan-Meier survival plot and the hazard ratio (HR) with $95 \%$ confidence intervals $(\mathrm{CI})$, and $\log$ rank $P$ value was calculated.

Survival validation of CENPA in GSE14520 and TCGA were conducted in Graphpad Prism v8.0 (GraphPad Software, CA, US).

2.3. Protein-Protein-Interaction Analysis. Protein-protein interaction analysis of CENPA was performed in online database STRING v11.0 (https://string-db.org/). The minimum required interaction score of parameters interacted with CENPA was set as the highest confidence $(\geq 0.9)$. 

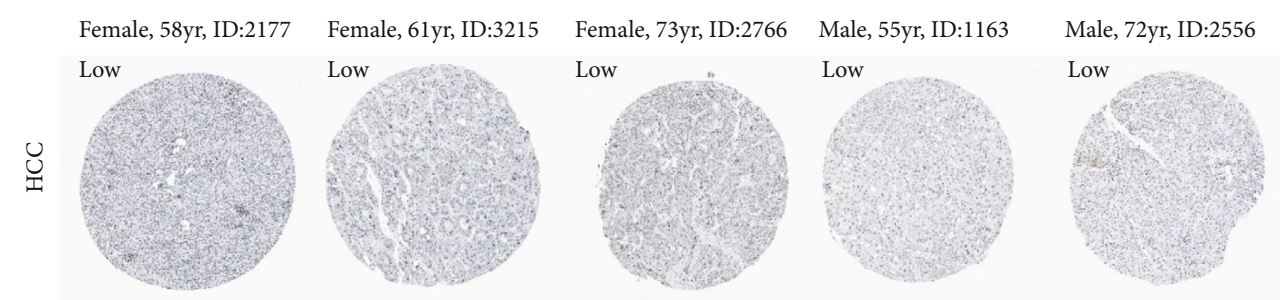

Male, $80 \mathrm{yr}$, ID:2280

(a)
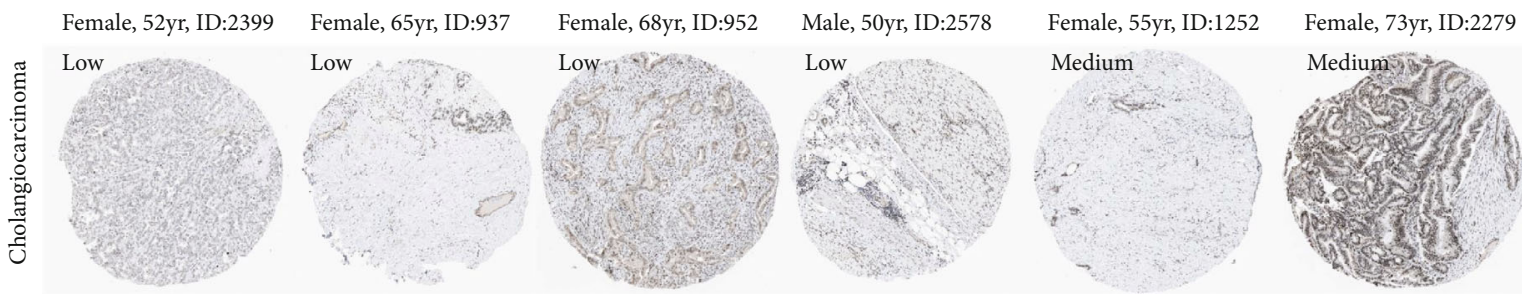

(b)

Figure 2: CENPA protein levels in HCC and cholangiocarcinoma detected by immunohistochemical assay in HPA database. (a) 6 HCC patients had low CENPA staining, (b) 4 cholangiocarcinoma patients had low CENPA staining, and 2 had medium staining.

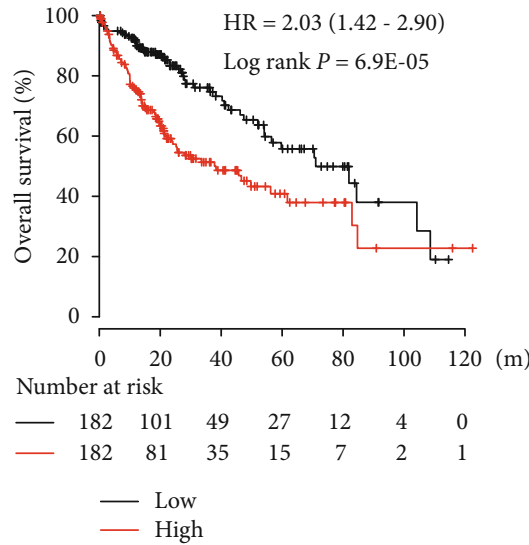

(a)

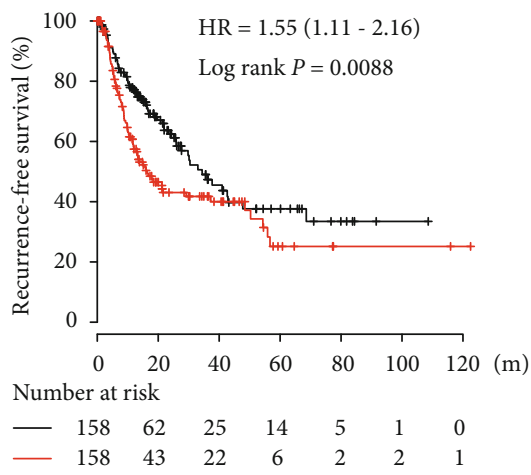

(c)

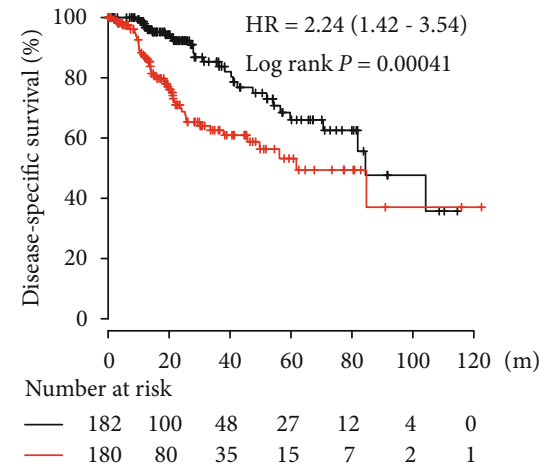

(b)

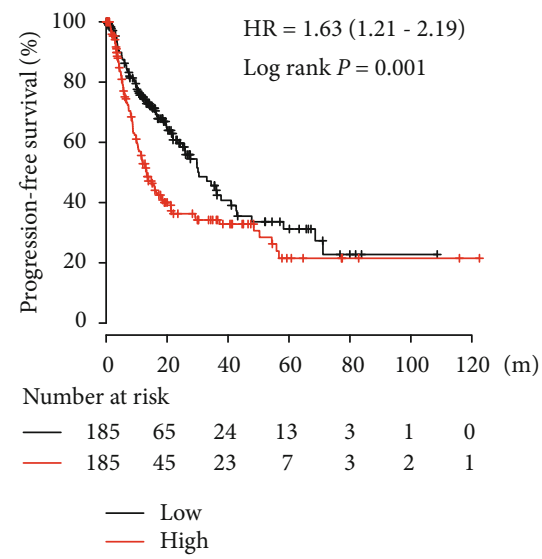

(d)

FIgure 3: (a) Overall survival, (b) disease-free survival, (c) recurrence-free survival, and (d) progression-free survival in hepatocellular carcinoma (HCC) patients grouped by CENPA mRNA median cutoff in Kaplan-Meier plotter. HCC patients with high CENPA mRNA levels in tumor tissues had worse overall survival (log rank $P=6.9 E-05)(a)$, disease-free survival (log rank $P=0.00041)(b)$, recurrencefree survival (log rank $P=0.0088)(\mathrm{c})$, and progression-free survival $(\log \operatorname{rank} P=0.001)(\mathrm{d})$. 

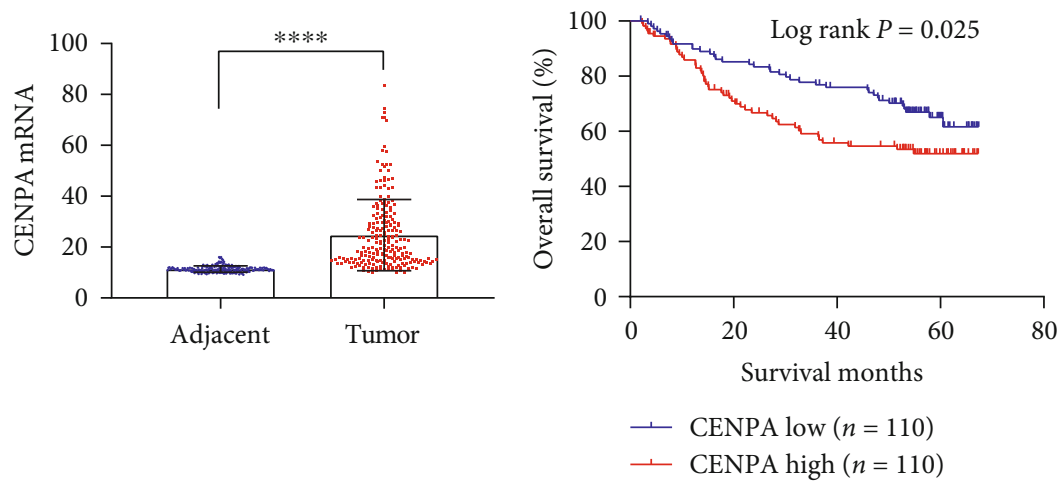

(a)

(b)

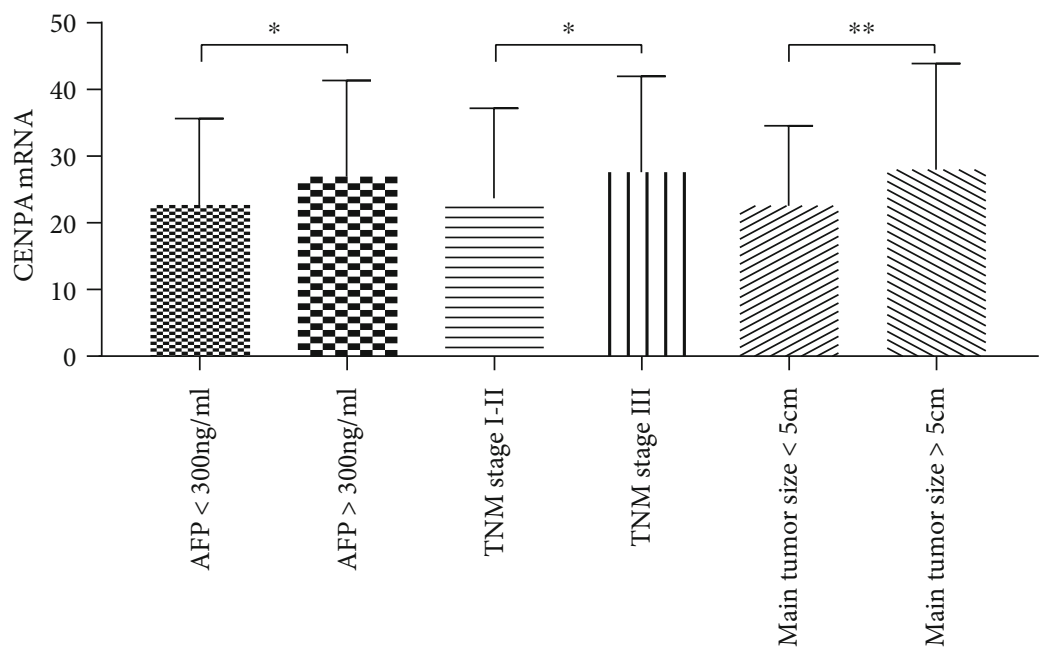

(c)

Figure 4: In GSE14520, (a) CENPA mRNA was significantly overexpressed in tumor tissues compared with that in adjacent tissues $(P<0.0001)$; (b) HCC patients with high CENPA mRNA had poorer overall survival compared to those with low CENPA mRNA (log rank $P=0.025$ ); and (c) CENPA mRNA was significantly higher in HCC patients with AFP > $300 \mathrm{ng} / \mathrm{ml}$, TNM stage III, and/or main tumor size $>5 \mathrm{~cm}($ all $P<0.05)$.

2.4. Cox Proportional Hazard Regression Model Establishment. Using the R program, the edgeR package [23] was used for identifying differentially expressed genes (DEGs) in HCC tumor and adjacent tissues, the survival package (https://cran .r-project.org/web/packages/survival/index.html) was used for conducting univariate and multivariate Cox regression analyses, and then, the Cox regression formula was calculated. According to this formula, HCC patients were divided into two groups: the high-risk group and the low-risk group. Survival analysis between these two groups was also performed by survival package. survivalROC (https://cran.rproject.org/web/packages/survivalROC/index.html) was used to perform a receiver operating characteristic (ROC) curve of the model for the prediction of HCC overall survival (OS). pheatmap package (https://cran.r-project.org/web/packages/ pheatmap/index.html) was used to summarize the gene expression of parameters in the Cox regression formula.

2.5. Statistical Analysis. Graphpad Prism v8.0 (GraphPad Software, CA, US) was used. Student's $t$-test or MannWhiney $U$ test was performed to analyze the differences of gene expression. Kaplan-Meier survival analysis was per- formed in GSE14520 and TCGA datasets. A two-tailed $P<0.05$ was considered significant.

\section{Results}

3.1. CENPA Expression. In TCGA, we obtained 50 paired tumor and nontumor tissues of HCC patients; the heatmap of the CENPA mRNA expression was shown in Figure 1(a), which indicated that CENPA mRNA was apparently upregulated in HCC tumors. When all microarray data of HCC patients in TCGA was included, CENPA mRNA was significantly overexpressed in tumor tissues compared with adjacent tissues $(P<0.0001$, Figure 1(b)). Consistently, CENPA mRNA were also significantly elevated in HCC tumors compared with those in nontumors in GEO series including GSE36376, GSE60502, GSE74656, and GSE77314 (all $P<0.01$, Figures 1(c)-1(f)).

In HPA database, we identified the CENPA protein expression in liver cancer. As shown in Figure 2, 6 HCC patients had low CENPA staining (Figure 2(a)), and 4 cholangiocarcinoma patients had low CENPA staining and 2 had medium staining (Figure 2(b)). Unfortunately, the 


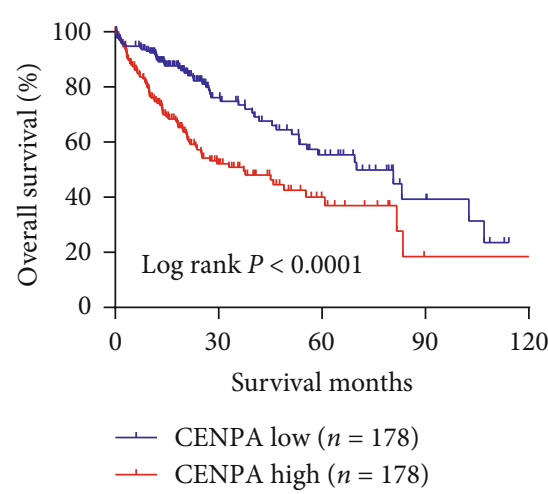

(a)

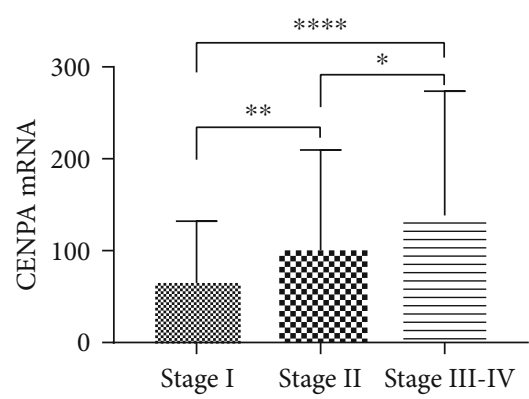

(c)

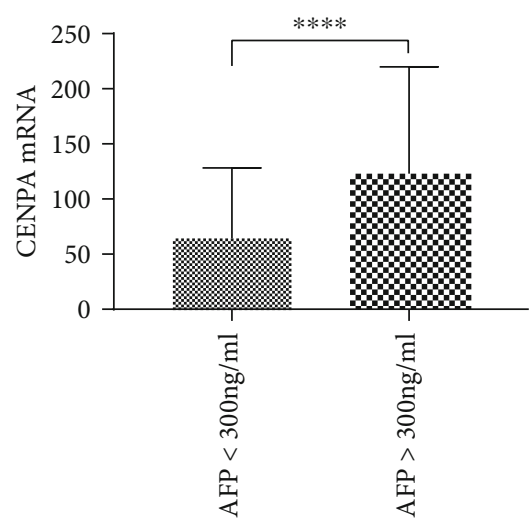

(e)

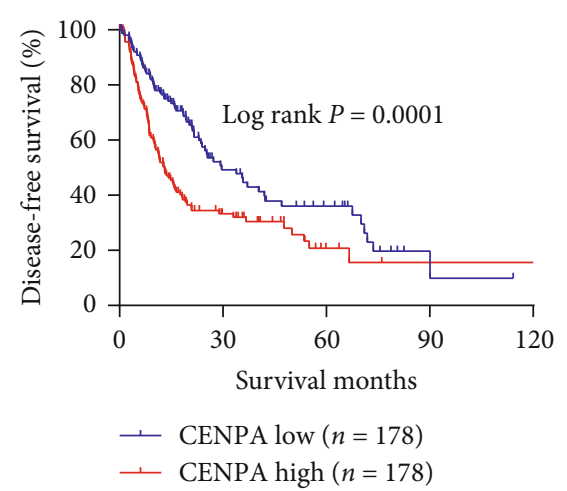

(b)

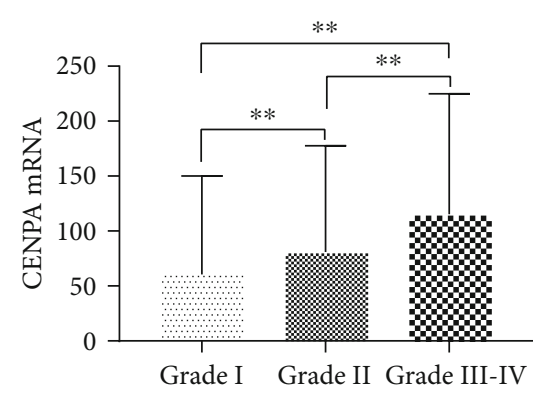

(d)

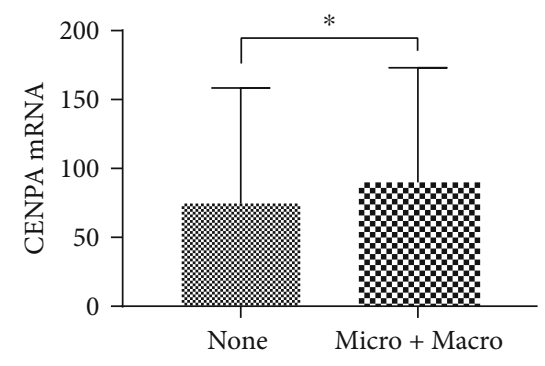

(f)

Figure 5: In TCGA dataset, HCC patients with high CENPA mRNA levels had (a) worse overall survival (log rank $P<0.0001)$ and (b) disease-free survival ( $\log \operatorname{rank} P=0.0001)$ compared to those with low CENPA mRNA; CENPA mRNA was significantly increased in HCC patients with (c) advanced AJCC stage (all $P<0.05)$, (d) advanced pathology grade (all $P<0.05)$, (e) AFP $>300 \mathrm{ng} / \mathrm{ml}(P<0.0001)$, and $(\mathrm{f})$ vascular invasion $(P<0.05)$.

CENPA protein expression in normal liver tissues was not available.

3.2. Associations between CENPA and HCC Survival. In the Kaplan-Meier plotter platform, CENPA mRNA overexpression was significantly correlated with overall survival (OS), disease-specific survival (DSS), recurrence-free survival (RFS), and progression-free survival (PFS) $(\mathrm{HR}=2.03$, $P=6.9 E-05 ; \mathrm{HR}=2.24, P=0.0041 ; \mathrm{HR}=1.55, P=0.0088$; and $\mathrm{HR}=1.63, P=0.001$, respectively, Figure 3 ).

3.3. Validation of CENPA in GSE14520 and TCGA. For validation, we included GSE14520 and TCGA datasets. In
GSE14520, CENPA mRNA was significantly upregulated in tumor tissues compared to adjacent tissues $(P<0.0001$, Figure 4(a)). High CENPA mRNA in tumors is significantly associated with poor OS in HCC patients $(P=0.025$, Figure 4(b)). In addition, CENPA mRNA overexpression was correlated with AFP elevation, advanced TNM stage, and larger tumor size (all $P<0.05$, Figure $4(\mathrm{c})$ ).

In the TCGA PanCancer Altas, CENPA mRNA upregulation was negatively associated with OS and DFS in HCC patients $(P<0.0001$ and $P=0.0001$, respectively, Figures 5(a) and 5(b)). Also, CENPA mRNA was increased in advanced AJCC stage (all $P<0.05$, Figure 5(c)) and high pathology grade (all $P<0.01$, Figure 5(d)). Moreover, 


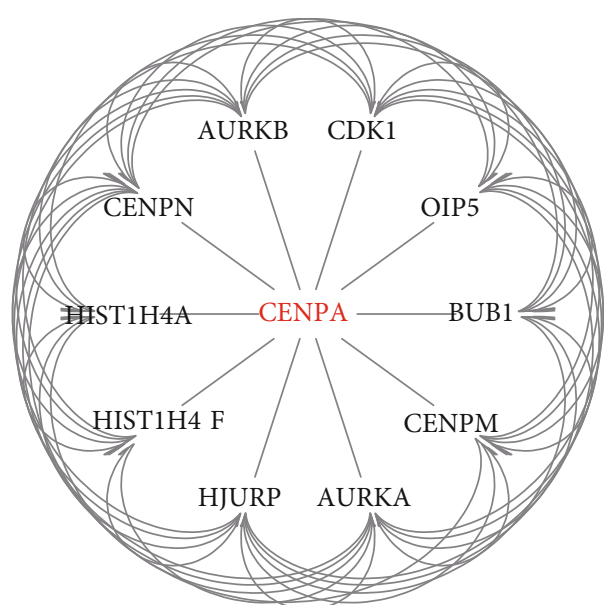

(a)

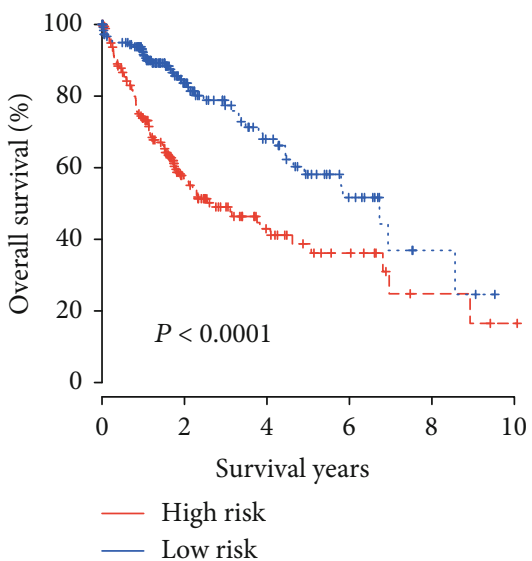

(c)

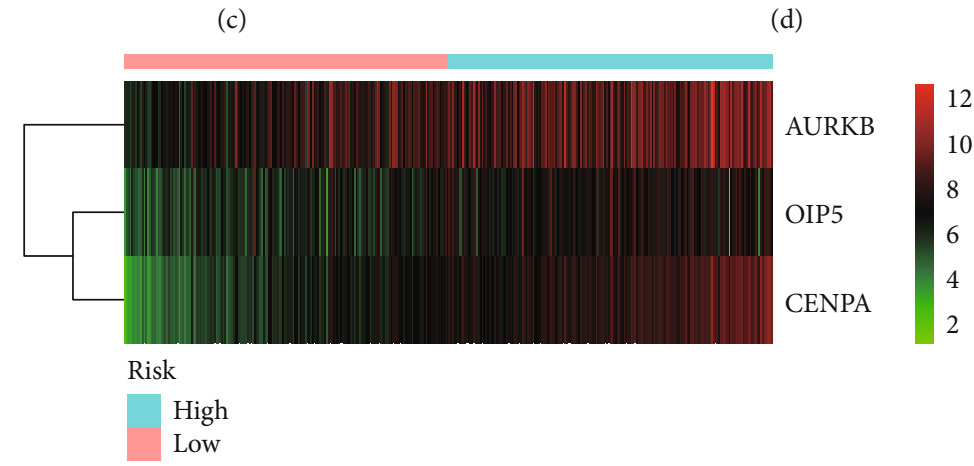

(e)

\begin{tabular}{lcc}
\hline Gene & Coefficient & $P$ value \\
\hline OIP5 & -0.14 & 0.0685 \\
AURKB & -0.21 & 0.0594 \\
CENPA & 0.57 & $2.89 \mathrm{e}-06$ \\
\hline$n=370$, number of events $=130$ \\
$P=2.02 \mathrm{e}-07$ \\
$y=-0.14 \times$ OIP5 $-0.21 \times$ AURKB $+0.57 \times$ CENPA
\end{tabular}

(b)

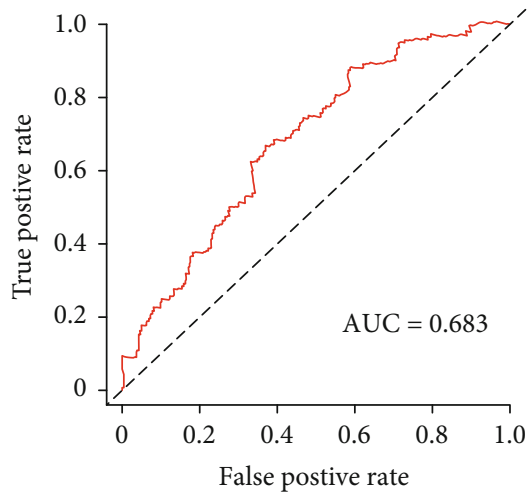

(d)

FIGURE 6: (a) Protein-protein interaction of CENPA in STRING; (b) multivariate Cox regression hazard model of CENPA interactive genes; (c) HCC patients with high risk in the Cox regression model had poorer overall survival than those with low risk (log rank $P<0.0001)$; (d) ROC curve of Cox regression model for overall survival in HCC patients; and (e) heatmap of genes included in the Cox regression model including CENPA, OIP5, and AURKB by risk scores.

CENPA mRNA was significantly elevated in HCC patients with a high AFP level and vascular invasion $(P<0.0001$ and $P<0.05$, respectively, Figures 5(e) and 5(f)).

3.4. Cox Regression Hazard Model Establishment. Using STRING online service, 10 genes including AURKB, CENPN, HIST1H4A, HIST1H4F, HJURP, AURKA, CENPM, BUB1, and OIP5 were interacted with CENPA (Figure 6(a)). In $\mathrm{R}$ program, univariate and multivariate Cox regression included 3 genes (CENPA, AURKB, and OIP5) in the Cox proportional hazard regression model, with a formula as $y=-0.14 \times$ OIP5 $-0.21 \times$ AURKB $+0.57 \times$ CENPA (Figure 6(b)). According to the formula, HCC patients were divided into the high-risk group and the low-risk group, with a median risk score. As shown in Figure 5(c), HCC patients with high risk had poor OS compared with low-risk patients $(P<0.0001$, Figure 6(c)). To identify the accuracy of this model for the prediction of OS in HCC patients, the ROC 
curve with an area under the curve (AUC) equal to 0.683 was summarized in Figure 6(d). The heatmap of CENPA, OIP5, and AURKB expression in high-risk and low-risk HCC patients was summarized in Figure 6(e).

\section{Discussion}

Altered expression of CENPA was frequently observed in many types pf human malignancies including lung cancer, colorectal cancer, breast cancer, and HCC [11, 24-27] and implicated in cell cycle regulation, cell survival, and genetic stability $[11,12,28,29]$. Our results from several datasets revealed that CENPA mRNA was significantly upregulated in HCC tumors, which is consistent with previous reports $[26,28]$. Using immunohistochemical analysis, Li et al. showed that the positive rate of CENPA in HCC samples was significantly higher than nonneoplastic liver tissues [26]. Considering both our results and the previous reports, we assumed that CENPA should be an oncogene in HCC progression.

The prognostic value of CENPA in human cancers was partly illustrated [11]. CENPA was an independent prognostic factor for lung adenocarcinoma, primary osteosarcoma, and epithelial ovarian cancer [24, 30-32]. High CENPA was significantly associated with advanced pathological grade, $\mathrm{pT}$ status, $\mathrm{pN}$ status, pleural invasion, high Ki-67 expression, and p53 positivity in lung adenocarcinoma [24, 32]. Similarly, CENPA upregulation was associated with tumor size, cancer recurrence, lung metastasis, high Ki-67 expression, and p53 positivity in primary osteosarcoma [30]. Additionally, CENPA elevation was significantly correlated with advanced pathology grade, tumor stage, and poor survival in human epithelial ovarian cancer [31]. CENPA was an independent predictor for relapse in estrogen receptor- (ER-) positive breast cancer patients not receiving systematic therapy but not in ER-negative patients. And CENPA also was not a prognostic marker of chemotherapy response [10]. Increased CENPA showed weak association with DFS in invasive breast cancer [27].

Unfortunately, few reports have focused on the clinical relationships between CENPA and HCC progression. A report by $\mathrm{Li}$ et al. found that CENPA overexpression was associated with advanced histological grade, positive serum HBsAg status, Ki-67 expression, and p53 immunopositivity [12]. Our results revealed that CENPA upregulation was correlated with poor outcomes (OS, DSS, RFS, and PFS) and progressive clinico-pathological features including AFP elevation, advanced tumor stage, vascular invasion, and tumor size in HCC patients, which were validated in both GSE14520 and TCGA datasets. Hence, we supposed that CENPA might play an oncogenic role in HCC progression. Previous basic experiment studies indicated that CENPA knockdown could reduce cell proliferation, block cell cycle at G1 phase, and induce apoptosis in HepG2 cells. Conversely, CENPA overexpression promoted cell growth and reduced apoptosis in HCC cell $[12,28]$. Additionally, $\mathrm{HBx}$ deletion was frequently observed in HBV-related HCC tissues. CENPA expression was positively associated with $\mathrm{HBx}$ mutation in HCC tissues. And $\mathrm{HBx}$ mutant increased
CENPA mRNA and protein expression remarkably compared to full-length HBx [13].

This report has some limitations. Firstly, the analysis was based on online bioinformatic analysis in transcriptional level, without CENPA protein data. Secondly, we could not perform experimental research for probing potential oncogenic mechanisms of CENPA in HCC development. Thirdly, this study had relatively small samples and performed within limited institute, and there was no follow-up data for our own from available HCC patients. Prospective studies with large sample should be performed to validate the results of this analysis. Even though we considered previous reports, we cautiously drew the hypothesis that CENPA overexpression contributed to unfavorable prognosis in HCC patients.

\section{Data Availability}

The data used to support the findings of this study are available from the corresponding author upon request.

\section{Conflicts of Interest}

The authors report no conflicts of interest in this work.

\section{Authors' Contributions}

Yuan Zhang and Lei Yang contributed equally to this work.

\section{Acknowledgments}

This work was supported by the National Natural Science Foundation of China (81803901), Medical Guidance Project of Shanghai Science and Technology Committee (19401931600), Shanghai Sailing Program (17YF1416000), and the Fundamental Research Funds for the Central Universities (2019-JYB-JS-047).

\section{References}

[1] M. M. Valdivia, K. Hamdouch, M. Ortiz, and A. Astola, "CENPA a genomic marker for centromere activity and human diseases," Current Genomics, vol. 10, no. 5, pp. 326335, 2009.

[2] E. V. Howman, K. J. Fowler, A. J. Newson et al., "Early disruption of centromeric chromatin organization in centromere protein A (Cenpa) null mice," Proceedings of the National Academy of Sciences of the United States of America, vol. 97, no. 3, pp. 1148-1153, 2000.

[3] P. Heun, S. Erhardt, M. D. Blower, S. Weiss, A. D. Skora, and G. H. Karpen, "Mislocalization of the Drosophila centromere-specific histone CID promotes formation of functional ectopic kinetochores," Developmental Cell, vol. 10, no. 3, pp. 303-315, 2006.

[4] R. L. Shrestha, G. S. Ahn, M. I. Staples et al., "Mislocalization of centromeric histone $\mathrm{H} 3$ variant CENP-A contributes to chromosomal instability (CIN) in human cells," Oncotarget, vol. 8, no. 29, pp. 46781-46800, 2017.

[5] W. Zhang, J. H. Mao, W. Zhu et al., "Centromere and kinetochore gene misexpression predicts cancer patient survival and response to radiotherapy and chemotherapy," Nature Communications, vol. 7, no. 1, article 12619, 2016. 
[6] W. Zhang, G. H. Karpen, and Q. Zhang, "Exploring the role of CENP-A Ser18 phosphorylation in CIN and tumorigenesis," Cell Cycle, vol. 16, no. 24, pp. 2323-2325, 2017.

[7] A. B. Sharma, S. Dimitrov, A. Hamiche, and E. Van Dyck, "Centromeric and ectopic assembly of CENP-A chromatin in health and cancer: old marks and new tracks," Nucleic Acids Research, vol. 47, no. 3, pp. 1051-1069, 2019.

[8] L. L. Sullivan, C. D. Boivin, B. Mravinac, I. Y. Song, and B. A. Sullivan, "Genomic size of CENP-A domain is proportional to total alpha satellite array size at human centromeres and expands in cancer cells," Chromosome Research, vol. 19, no. 4, pp. 457-470, 2011.

[9] R. K. Athwal, M. P. Walkiewicz, S. Baek et al., "CENP-A nucleosomes localize to transcription factor hotspots and subtelomeric sites in human cancer cells," Epigenetics \& Chromatin, vol. 8, no. 1, 2015.

[10] S. L. McGovern, Y. Qi, L. Pusztai, W. F. Symmans, and T. A. Buchholz, "Centromere protein-A, an essential centromere protein, is a prognostic marker for relapse in estrogen receptor-positive breast cancer," Breast Cancer Research, vol. 14, no. 3, p. R72, 2012.

[11] X. Sun, P. L. Clermont, W. Jiao et al., "Elevated expression of the centromere protein-A (CENP-A)-encoding gene as a prognostic and predictive biomarker in human cancers," International Journal of Cancer, vol. 139, no. 4, pp. 899-907, 2016.

[12] Y. Li, Z. Zhu, S. Zhang et al., "ShRNA-targeted centromere protein A inhibits hepatocellular carcinoma growth," PLoS One, vol. 6, no. 3, article e17794, 2011.

[13] L. Liu, Y. Li, S. Zhang, D. Yu, and M. Zhu, "Hepatitis B virus X protein mutant upregulates CENP-A expression in hepatoma cells,” Oncology Reports, vol. 27, no. 1, pp. 168-173, 2012.

[14] S. Roessler, H. L. Jia, A. Budhu et al., "A unique metastasis gene signature enables prediction of tumor relapse in early-stage hepatocellular carcinoma patients," Cancer Research, vol. 70, no. 24, pp. 10202-10212, 2010.

[15] S. Roessler, E. L. Long, A. Budhu et al., "Integrative genomic identification of genes on $8 p$ associated with hepatocellular carcinoma progression and patient survival," Gastroenterology, vol. 142, no. 4, pp. 957-966.e12, 2012, e912.

[16] P. J. Thul, L. Åkesson, M. Wiking et al., "A subcellular map of the human proteome," Science, vol. 356, no. 6340, article eaal3321, 2017.

[17] M. Uhlen, L. Fagerberg, B. M. Hallstrom et al., "Tissue-based map of the human proteome," Science, vol. 347, no. 6220, 2015.

[18] M. Uhlen, C. Zhang, S. Lee et al., "A pathology atlas of the human cancer transcriptome," Science, vol. 357, no. 6352, 2017.

[19] E. Cerami, J. Gao, U. Dogrusoz et al., "The cBio cancer genomics portal: an open platform for exploring multidimensional cancer genomics data," Cancer Discovery, vol. 2, no. 5, pp. 401404, 2012.

[20] J. Gao, B. A. Aksoy, U. Dogrusoz et al., "Integrative analysis of complex cancer genomics and clinical profiles using the cBioPortal," Science Signaling, vol. 6, no. 269, 2013.

[21] O. Menyhárt, A. Nagy, and B. Győrffy, "Determining consistent prognostic biomarkers of overall survival and vascular invasion in hepatocellular carcinoma," Royal Society Open Science, vol. 5, no. 12, article 181006, 2018.

[22] Á. Nagy, A. Lánczky, O. Menyhárt, and B. Győrffy, "Validation of miRNA prognostic power in hepatocellular carcinoma using expression data of independent datasets," Scientific Reports, vol. 8, no. 1, p. 9227, 2018.

[23] M. D. Robinson, D. J. McCarthy, and G. K. Smyth, “edgeR: a bioconductor package for differential expression analysis of digital gene expression data," Bioinformatics, vol. 26, no. 1, pp. 139-140, 2010.

[24] Q. Wu, Y. M. Qian, X. L. Zhao et al., "Expression and prognostic significance of centromere protein $\mathrm{A}$ in human lung adenocarcinoma," Lung Cancer, vol. 77, no. 2, pp. 407-414, 2012.

[25] T. Tomonaga, K. Matsushita, S. Yamaguchi et al., "Overexpression and mistargeting of centromere protein-A in human primary colorectal cancer," Cancer Research, vol. 63, no. 13, pp. 3511-3516, 2003.

[26] Y. M. Li, X. H. Liu, X. Z. Cao, L. Wang, and M. H. Zhu, "Expression of centromere protein A in hepatocellular carcinoma," Zhonghua Bing Li Xue Za Zhi, vol. 36, no. 3, pp. 175-178, 2007.

[27] A. B. Rajput, N. Hu, S. Varma et al., "Immunohistochemical assessment of expression of centromere protein-A (CENPA) in human invasive breast cancer," Cancers, vol. 3, no. 4, pp. 4212-4227, 2011.

[28] Y. M. Li, Z. Zhu, Y. Chen, Z. G. Luo, M. Shi, and M. H. Zhu, "Effect of siRNA targeting centromere protein-A gene on biological behavior of HepG2 cells," Zhonghua Bing Li Xue Za Zhi, vol. 37, no. 2, pp. 124-128, 2008.

[29] J. Behnan, Z. Grieg, M. Joel, I. Ramsness, and B. Stangeland, "Gene knockdown of CENPA reduces sphere forming ability and stemness of glioblastoma initiating cells," Neuroepigenetics, vol. 7, pp. 6-18, 2016.

[30] X. M. Gu, J. Fu, X. J. Feng et al., "Expression and prognostic relevance of centromere protein A in primary osteosarcoma," Pathology, Research and Practice, vol. 210, no. 4, pp. 228233, 2014.

[31] J. J. Qiu, J. J. Guo, T. J. Lv et al., "Prognostic value of centromere protein-A expression in patients with epithelial ovarian cancer," Tumour Biology, vol. 34, no. 5, pp. 2971-2975, 2013.

[32] W. T. Liu, Y. Wang, J. Zhang et al., "A novel strategy of integrated microarray analysis identifies CENPA, CDK1 and CDC20 as a cluster of diagnostic biomarkers in lung adenocarcinoma," Cancer Letters, vol. 425, pp. 43-53, 2018. 